\section{Capgras Syndrome in a Patient With Multiple Sclerosis: A Case Report}

To the Editor: Capgras syndrome, or the delusion of doubles, is a relatively rare phenomenon, occurring predominantly in the context of schizophrenia and affective disorders, although $25 \%$ to $40 \%$ of cases are associated with disorders such as dementia, epilepsy, and cerebrovascular disease. ${ }^{1}$ We present a patient with multiple sclerosis (MS) who developed Capgras syndrome during an acute relapse.

Case report. Ms A, a 45-year-old woman, was brought to the emergency room (ER) in January 2007 by her husband after she assaulted him. While in the ER, she reported that her husband was not really her husband, that her neighbors had keys to her house, and that they had raped her and wanted to kill her. She also believed that her teenage sons had been murdered and the 2 males living in her home were strangers.

Ms A had been diagnosed with bipolar disorder 26 years ago and had 1 previous psychiatric admission. She had taken risperidone and oxcarbazepine in the past but not recently. She had also been diagnosed with MS 20 years previously and was being closely followed by a neurologist but was taking no medications.

Mental status examination revealed a disheveled woman who made fair eye contact. She had fluent but mildly aprosodic speech. Her mood was fair and her affect constricted. Her thought process was illogical but without loose associations or flight of ideas. Her thought content was significant only for delusions.

Neurologic examination revealed bilateral optic disc pallor consistent with a previous optic neuritis. A magnetic resonance imaging scan of the head showed a single enhancing lesion in the right frontal lobe periventricular region, suggesting acute disease, and diffuse lesions in the temporal lobes and subcortically. Lumbar puncture revealed a positive MS panel with an elevated cerebrospinal fluid index at 1.31 , elevated albumin at $38.1 \mathrm{~g} / \mathrm{dL}$, and 4 oligoclonal bands. Neuropsychological testing revealed mild cognitive impairment deemed consistent with subcortical pathology.

She initially refused food and medication, as she believed that someone was trying to poison her. Aripiprazole $10 \mathrm{mg} / \mathrm{d}$ was initiated 10 days after admission and was increased to $15 \mathrm{mg} / \mathrm{d}$ after 2 days. Within a few days, Ms A started eating and became more pleasant but still fixated on her delusions. Considering this to be a relapse of MS, she was offered methylprednisolone, which she refused. However, she agreed to intravenous immunoglobulin 26 days into her admission. She gradually became more realistic about her family's identity and denied that her family members were imposters. Upon discharge, she was less delusional and acknowledged that her family would be at home when she got there.

Capgras syndrome, originally described by Capgras and Reboul-Lachaux ${ }^{2}$ in 1923 , consists of a delusional belief that a person has been replaced by imposters, look-alikes, or doubles. Neuroimaging studies have linked Capgras syndrome to abnormalities in the frontotemporal regions, with some authors proposing a deep right frontal lesion disconnecting it from the temporal and limbic areas with a subsequent disturbance in a sense of familiarity of people and places. ${ }^{3}$ Alternatively, it has been suggested that the dorsal pathway between the visual cortex and the limbic system via the inferior parietal lobule is disconnected in this syndrome. ${ }^{4}$ The only reported case of Capgras syndrome in MS showed hypoperfusion in the right parietal cortex on single photon emission computed tomography (SPECT). ${ }^{1}$

Despite our patient's history of bipolar disorder, her current presentation was not consistent with mania, and we feel that an acute relapse of MS triggered this episode of psychosis. On the basis of the response of this patient, we suggest that patients with an acute relapse of MS presenting with a co-occurring delusional disorder might be best managed by treating the underlying relapse of MS combined with an antipsychotic medication. If steroids are considered for use in this setting, careful monitoring is required to avoid exacerbation of the psychotic episode, especially if the patient has a diagnosis of bipolar disorder.

\section{REFERENCES}

1. Lebert F, Pasquier F, Steinling M, et al. SPECT data in a case of secondary Capgras delusion. Psychopathology. 1994;27 (3-5):211-214.

2. Capgras J, Reboul-Lachaux J. Illusion des sosies dans un délire systématisé chronique. Bulletin de la Société Clinique de Médicine Mentale. 1923;2:6-16.

3. Alexander MP, Stuss DT. Capgras syndrome: a reduplicative phenomenon. J Psychosom Res. 1998;44(6):637-639.

4. Edelstyn NM, Oyebode F. A review of the phenomenology and cognitive neuropsychological origins of the Capgras syndrome. Int J Geriatr Psychiatry. 1999;14(1):48-59.

Ashish Sharma, MD

Mariam Garuba, MD

MariamGaruba@creighton.edu

Matthew Egbert, MD

Author affiliations: Department of Psychiatry, University of Nebraska Medical Center (all authors); and Department of Psychiatry, Creighton University (Dr Garuba), Omaha, Nebraska. Financial disclosure: None reported. Funding/support: None reported.

doi:10.4088/PCC.08100702

(C) Copyright 2009 Physicians Postgraduate Press, Inc. 